\title{
Progress Towards On-chip Single Photon Sources Based on Colloidal Quantum Dots in Silicon Nitride Devices
}

\author{
Suzanne Bisschop ${ }^{1,2,3}$, Yunpeng $\mathrm{Zhu}^{2,3}$, Weiqiang $\mathrm{Xie}^{2,3}$, Antoine Guille ${ }^{1,3}$, Zeger Hens ${ }^{1,3}$, Dries Van \\ Thourhout $^{2,3}$, Edouard Brainis ${ }^{1,3}$ \\ ${ }^{1}$ Physics and Chemistry of Nanostructures, Ghent University, Krijgslaan 281-S3, 9000 Ghent, Belgium \\ ${ }^{2}$ Photonics Research Group, INTEC Department, Ghent University-IMEC, Sint-Pietersnieuwstraat 41, 9000 Ghent, Belgium \\ ${ }^{3}$ Center for Nano and Biophotonics (NB Photonics), Ghent University, B-9000 Ghent, Belgium \\ Author e-mail address: (edouard.brainis@ugent.be)
}

\begin{abstract}
New results on integration of colloidal quantum dots (QDs) into SiN microstructures are reported, including QD positioning with nanometric accuracy and the efficient coupling of their emission to waveguides and cavities. The results are relevant to on-chip quantum optics and information processing.

OCIS codes: (270.0270) Quantum Optics; (220.4000) Microstructure fabrication.
\end{abstract}

\section{Introduction}

In the beginning of the years 2000, "optical quantum computing" [1] has emerged as a new and very promising paradigm to demonstrate that quantum computers have the potential to radically outperform classical digital computers. Optical quantum computation is appealing because it requires only single-photon sources, linear optical elements and single-photon detectors. In addition it has been shown that fault tolerant computing is possible if the product of the single-photon emission efficiency $\eta_{S}$ and the single-photon detection efficiency $\eta_{D}$ satisfies $\eta_{\mathrm{S}} \times \eta_{\mathrm{D}}>2 / 3$, and all other optical components operate perfectly [2].

At visible wavelengths, $\mathrm{Si}$ avalanche photodiodes (APDs) reach detection efficiencies as high as $\eta_{\mathrm{D}}=80 \%$. The linear optical networks (made of beam-splitters, phase-shifters, etc.) needed for optical quantum computing can be with table-top optical components or fiber optics. These approaches offer high flexibility but suffer from thermal and mechanical instabilities that hinder their scalability. Complex optical networks are best realized using integrated photonics devices on a microchip. Many recent experiments successfully validated this approach by performing quantum optics experiments with photons at $800 \mathrm{~nm}$ (a wavelength at which Si APDs are still very efficient) propagating in integrated photonic circuits $[3,4]$. Nowadays, the major technological obstacle to optical quantum computing is the lack of efficient (high $\eta_{\mathrm{S}}$ ) sources of indistinguishable photons at wavelengths compatible with $\mathrm{Si}$ APDs. Single photons are always generated by parametric down-conversion, which is an inefficient, probabilistic (the photons are heralded) and therefore unscalable method.

In this work, we study the possibility to integrated triggered solid-state single photon emitters directly on a photonic chip. We specifically investigate photonic chips made of silicon nitride $\left(\mathrm{Si}_{3} \mathrm{~N}_{4}\right)$ because this material has a larger refractive index than silica $(n=2)$, while is highly transparent in the visible and near-infrared range. $\mathrm{Si}_{3} \mathrm{~N}_{4}$ devices and circuits can be realized using standard CMOS compatible processing technology on a SOI chip. Most importantly, the technology offers the possibility to embed alien solid-state single-photon emitters (such as colloidal quantum dot or diamond nanoparticles) inside the $\mathrm{Si}_{3} \mathrm{~N}_{4}$ host.

The major challenges consist in (i) deterministically positioning quantum emitters at predefined sites, (ii) simultaneously exciting a selected number of emitters and (iii) efficiently coupling the emitted single photons to a waveguide. Here, we discuss the problems (i) and (iii), since an elegant solution to the problem (ii) has been already proposed elsewhere [5]. Our solution to the problem (i) is restricted to the integration of colloidal quantum dots (QDs), while our solutions to the problem (iii) apply to any single photon emitting nanoparticle.

\section{Coupling single-photons to photonic circuits}

We analyze the coupling of single-photons emitted by single QDs to $\mathrm{Si}_{3} \mathrm{~N}_{4}$ waveguides using FDTD simulations. For rectangular strip waveguides, we find no significant Purcell enhancement of the emission (Purcell factors ranging between 0.7 and 1.3 for all dipole polarizations and little wavelength dependence). The coupling of the light to the waveguide is nevertheless high. For instance, for a $270 \times 300 \mathrm{~nm}$ strip waveguide, we find a $58 \%$ coupling efficiency for a central emission wavelength of $650 \mathrm{~nm}$ and a randomly oriented dipole moment.

The polarization of the emitted photons can be better controlled by embedding the QD into a thin $\mathrm{SiO}_{2}$ layer in the center of the $\mathrm{Si}_{3} \mathrm{~N}_{4}$ waveguide (see inset of Fig. 1a). FDTD simulations show a significant Purcell enhancement of spontaneous emission in the polarization orthogonal to the $\mathrm{SiO}_{2}$ layer. Fig. 1a shows the calculated Purcell factor 
$F_{i}$ in a $(150 \mathrm{~nm} \times h) \mathrm{Si}_{3} \mathrm{~N}_{4}$ waveguide with a $10-\mathrm{nm} \mathrm{SiO}_{2}$ layer as a function of $h$ and dipole orientation $\boldsymbol{n}_{i}$. For an otherwise isotropic emitter, the probability to emit a $i$-polarized photon is equal to $p_{i}=F_{i} /\left(\sum_{i} F_{i}\right)$. For $h=310 \mathrm{~nm}$, we find that $65 \%$ of the emitted photons have a polarization orthogonal to the $\mathrm{SiO}_{2}$ layer, $23 \%$ in the direction orthogonal to the waveguide, and $13 \%$ in the remaining direction. The emitted photons have an overall probability as high as $63 \%$ to be coupled into a guided mode. The photons emitted with a polarization orthogonal to the waveguide are not guided at all. Among the guided photons, $87.5 \%$ have a polarization orthogonal to the $\mathrm{SiO}_{2}$ layer and only $12.5 \%$ have the complementary polarization. This shows that strongly polarized photons can be coupled to waveguides with high efficiency, even at room temperature. Although, a photon emitted in a waveguide is in a superposition of two opposite propagation direction, a directional emission can be easily obtained using reflectors of one end of the waveguide or by placing the emitting QD in a Sagnac loop as shown in the Fig. $1 \mathrm{~b}$.

We also simulated QDs in free-standing $\mathrm{Si}_{3} \mathrm{~N}_{4}$ microdisk cavities. Such cavities can be manufactured with a high degree of reproducibility, can be vertically coupled to buried waveguides [6], and support high-Q modes. At cryogenic operation, QDs can have a spectrum as narrow as $5 \mathrm{GHz}(20 \mu \mathrm{eV}, 6.5 \mathrm{pm})$. In a $5-\mu \mathrm{m}$ disk, we calculated that a Q-factor of 2000 would be enough to get a Purcell factor of 9 and an extraction efficiency of $90 \%$ into the coupled waveguide. We report on the fabrication of such cavities (see Fig. 1c) and are currently characterizing them. For room temperature operation, smaller cavities are required if single-mode emission is desirable. We simulated that a free spectral range of about $40 \mathrm{~nm}$ can be achieved in $2 \mu \mathrm{m}$-sized $\mathrm{Si}_{3} \mathrm{~N}_{4}$ microdisk cavities and that the probability that the QD emit into the desired mode in such a cavity is $15 \%$ at room temperature.

\section{Positioning QDs with nanometric accuracy}

To efficiently couple single QD to waveguides or cavities, deterministic methods for positioning QDs at desired sites are needed. Here, we report on a novel and promising technique to achieve that goal. The technique combines substrate e-beam pattering, Langmuir-Blodgett deposition of a QD monolayer and site-selective lift-off. Fig. 1d shows that QD-patches of $62 \mathrm{~nm}$ are achievable. By mixing different types of QDs, it is possible to achieve a single optically active QD per patch.

\section{Conclusion}

In this work, we report on recent progress towards the efficient generation of single photons on a photonic chip. The techniques that we develop and combine are suitable for operating at room temperature as well as at cryogenic temperatures depending on the degree of coherence required for the photons. Simulations support the idea that single-photon sources with an efficiency $\eta_{\mathrm{S}}>2 / 3$ can be realized using colloidal QD in $\mathrm{Si}_{3} \mathrm{~N}_{4}$ microstructures.
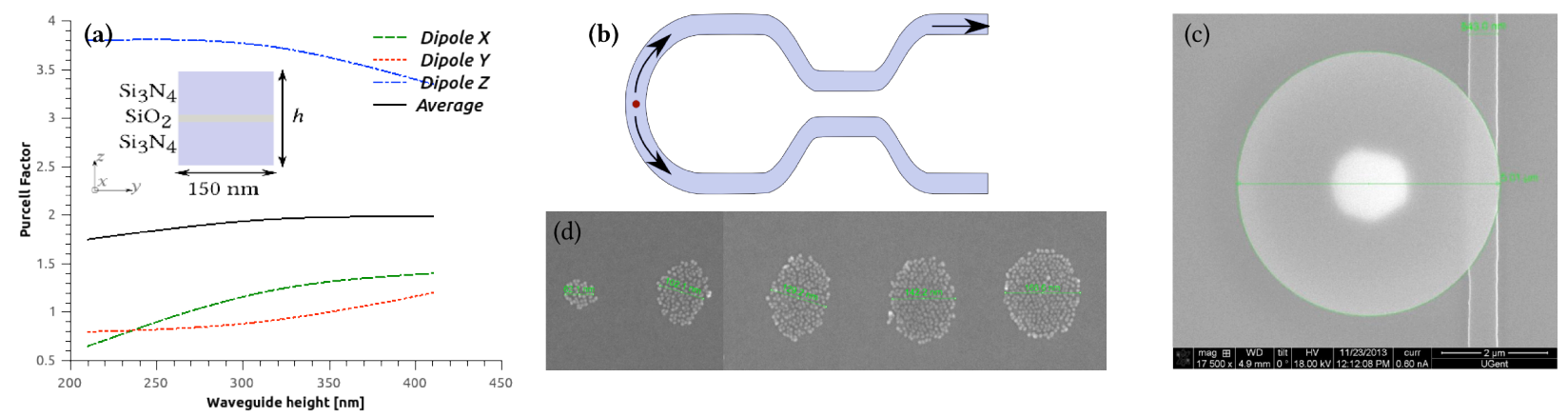

Figure 1: (a) Spontaneous emission enhancement factor for a single photon emitter embedded in $\mathrm{Si}_{3} \mathrm{~N}_{4}$ waveguide with a thin $\mathrm{SiO}_{2}$ layer. (b) Single photon self-interference makes it possible to collect all the emission in one output arm. (c) Fabricated 5 $\mu$ mmicrodisks coupled to a buried waveguide. (d) Positioning QDs with nanometric accuracy.

\section{References}

[1] E. Knill et al, "A scheme for efficient quantum computation with linear optics," Nature 409, 46-52 (2001).

[2] M. Varnava et al, "How good must single photon sources and detectors be for efficient linear optical quantum computation?" Phys. Rev. Lett. 100, $060502(2008)$

[3] A. Crespi et al, "Integrated multimode interferometers with arbitrary designs for photonic boson sampling," Nature Photon. 7, 545-549 (2013)

[4] J. W. Silverstone et al, "On-chip quantum interference between silicon photon-pair sources," Nature Photon. Advanced Online Publication (15 Dec. 2013)

[5] E. Brainis, "Towards parallel control of multiple single-photon emitters," in 2011 Conference on Lasers and Electro-Optics and 12th European Quantum Electronics Conference (2011).

[6] M. Ghulinyan, "Monolithic whispering-gallery mode resonators with vertically coupled integrated bus waveguides," IEEE Photon. Technol. Lett. 23, 1166-1168 (2011). 